\title{
Numerical Study on the Strength of Pipe Joints
}

\author{
Dai-Heng Chen and Kenichi Masuda \\ Department of Mechanical Engineering, Tokyo University of Science, Kagurazaka 1-3, Shinjuku-ku, Tokyo 162-8601, Japan \\ Correspondence should be addressed to Dai-Heng Chen, chend@rs.kagu.tus.ac.jp
}

Received 6 July 2011; Accepted 18 August 2011

Academic Editors: D. Aggelis, A. E. Huespe, and D. Zhou

Copyright ( $) 2011$ D.-H. Chen and K. Masuda. This is an open access article distributed under the Creative Commons Attribution License, which permits unrestricted use, distribution, and reproduction in any medium, provided the original work is properly cited.

\begin{abstract}
With respect to the collapse behavior of joints in a steel pipe truss structure, in the present study, we carry out numerical analyses using a general-purpose FEM software package on the yield strength and the stress distribution near the connection in order to obtain information on the collapse behavior and the yield strength of joints. $T$-joint and $Y$-joint are investigated. It is found that the joint strength is proportional to the $\lambda$ th power of thickness $T$ of a main tube for various joints with a fixed branch-to-chord diameter ratio $d / D$. The index $\lambda$ changes with $d / D$ and in the range of $d / D=0.1$ to $0.91, \lambda \cong 1.93$ to 1.55 holds with smaller values corresponding to larger $d / D$. A compressive axial force along the main tube will reduce the joint strength, and it is also found that the strength-reduction ratio for $Y$-joint is almost the same as that for $T$-joint having the same $T$ and $d / D$. Also, the joint strength is influenced by the span length of the main tube. The joint strength decreases as the span length increases. However, the influence is small for small $d / D$.
\end{abstract}

\section{Introduction}

Steel pipe truss structures are widely used in marine structures and large space structures, and the yield strength of joints in a steel pipe truss structure has been extensively studied [1-5]. A book entitled the "Recommendations for the Design and Fabrication of Tubular Truss Structures in Steel," published by Architectural Institute of Japan, is available for practical design [1]. However, although there are some studies based on numerical FEM analysis among these past works [6], most studies are purely experimental, and the proposed design equations are based on limited experimental conditions. Therefore, with respect to the collapse behavior of joints in a steel pipe truss structure, in the present study, we carry out numerical analyses using a generalpurpose FEM software package on the yield strength and the stress distribution near the connection in order to obtain information on the collapse behavior and the yield strength of joints.

\section{Numerical Analysis Method}

In this study, assuming an analysis model for the geometry of the $T$ - and $Y$-joints as shown in Figure 1, large-deformation elastic-plastic FEM analysis was carried out by using the general-purpose FEM software package, MSC. Marc on the deformation behavior of a joint assuming that compressive load is applied to the branch tube in the axial direction. The boundary condition is such that the compressive load is applied from above by a moving rigid wall in contact with the branch tube, while both ends of the main tube are simply supported.

Furthermore, structural steel pipes are the assumed material, and the stress-strain relation is assumed to obey the bilinear hardening rule. Unless otherwise noted, the material constants are set as follows: yield stress $\sigma_{Y}=360 \mathrm{MPa}$, Young's modulus $E=205 \mathrm{GPa}$, Poisson's ratio $\nu=0.3$, and strain-hardening coefficient $E_{h}=0.01 E$. The geometry parameters of the joint are span length $L / D=5$, main tube diameter $D=318.5 \mathrm{~mm}$, and common thickness of branch and main tubes $t=T=4.5 \mathrm{~mm}$.

In the finite element modeling, the size of the element is assumed to be about $20 \times 20 \mathrm{~mm}^{2}$ so that the deformation proceeds smoothly, and discretization is performed using 4-node quadrilateral thick shell finite elements. In this analysis, to model nonlinear behavior, the updated Lagrange method is used to consider geometrical nonlinearity, and 


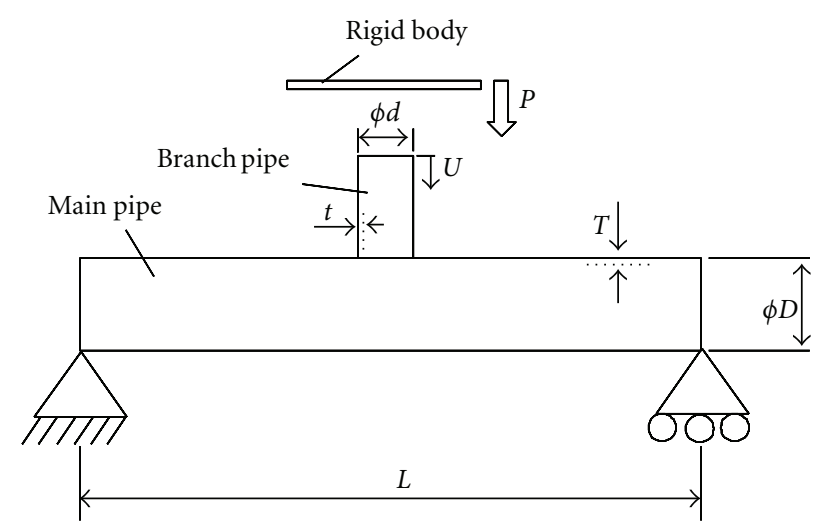

(a) $T$-joint

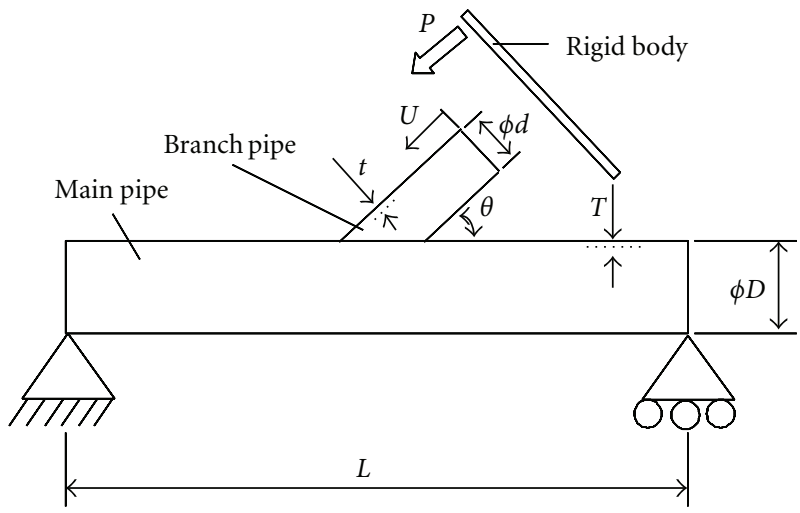

(b) $Y$-joint

Figure 1: Geometry of pipe joint.

the algorithm based on the Newton-Raphson method and the return-mapping method is used to solve the nonlinear equation.

\section{Analytical Results and Discussions}

3.1. Crush Behavior of a T-joint. Figure 2 shows the relation between the compressive load $P$ of a branch tube and displacement $U$, as evaluated for a $T$-joint with a branchto-main diameter ratio of $d / D=0.44$. The figure shows that load $P$ initially increases with compression displacement $U$ and shows a peak at a certain level of displacement. As known from the deformation behavior before and after the load peak shown in the figure, we have a compressive load peak because of the denting deformation on the surface of a cylindrical main tube. In addition, Figure 3 shows the contour diagram of von Mises stress distribution near the branch joint, where the brighter color denotes higher stress (the yellow color denotes the highest stress). The schematic view in Figure 3 shows the relation between each contour diagram and joint. Figure 3 also shows the contour diagram of von Mises stress distribution of the main tube at peak load for different branch-to-main diameter ratios (for the ratios of $d / D=0.1$ and $d / D=0.26$ ). As the figure shows, if $d / D$ is small (e.g., $d / D=0.1$ ), the largest stress appears at both ends of the branch tube (both ends of the branch tube as viewed from the axial direction of the main tube; since they are at the side surface of the main tube, we call them the "side" area). If $d / D$ is large (e.g., $d / D=0.44$ ), the largest stress appears before and after the branch tube (before and after the branch tube as viewed from the axial direction of the main tube; since they are at the uppermost parts of the main tube, we call them the "top" area).

Figure 2 also shows the experimental values obtained by other researchers [2]. As the figure shows, the experimental values and the numerical results of FEM analysis exhibit similar trends; therefore, it is confirmed that experimental results can be reproduced by the numerical simulation.

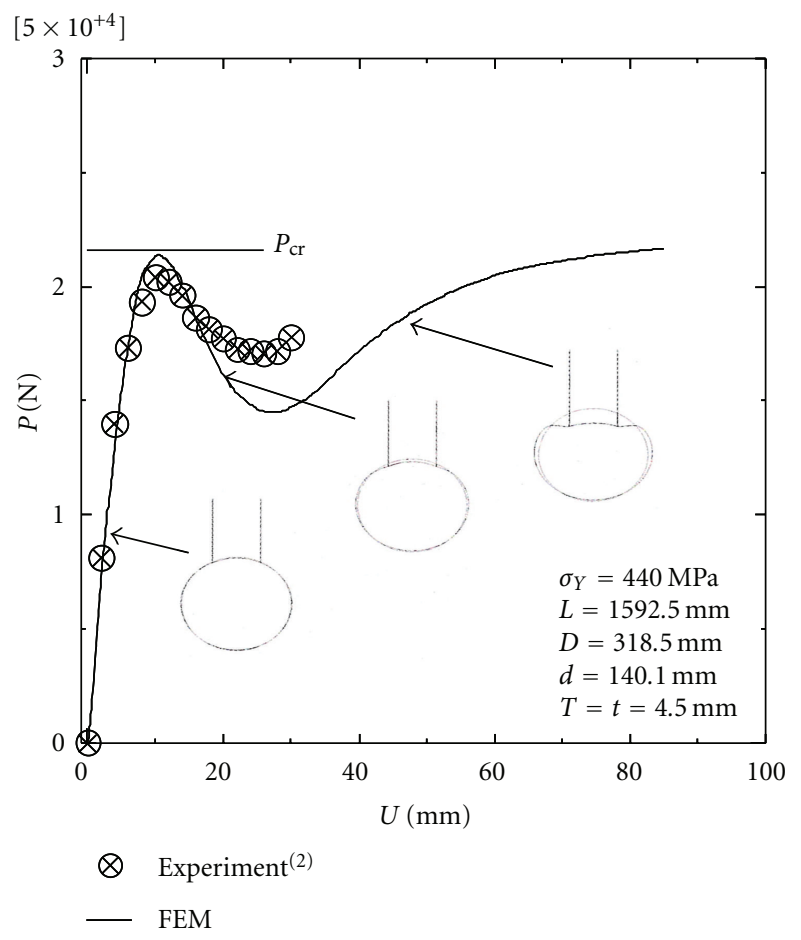

Figure 2: Relation between compressive load $P$ and displacement $U$ for a $T$-joint.

In the following discussion, the collapse load corresponding to the peak in the load-displacement curve shall be defined as the yield strength $P_{\text {cr }}$ of the said joint.

Figure 4 shows the relation between the yield strength of joint $P_{\mathrm{cr}}$ and $d / D$, with the thickness $T$ of the main tube as a parameter. In this figure, the vertical axis is the ratio of yield strength $P_{\mathrm{cr}}$ and $\left(\sigma_{Y} T^{2}\right): P_{\mathrm{cr}} / \sigma_{Y} T^{2}$. As shown in the figure, the ratio $P_{\mathrm{cr}} / \sigma_{Y} T^{2}$ is mainly a function of $d / D$, increasing with $d / D$ but also depends on the thickness $T$ of the main tube. 


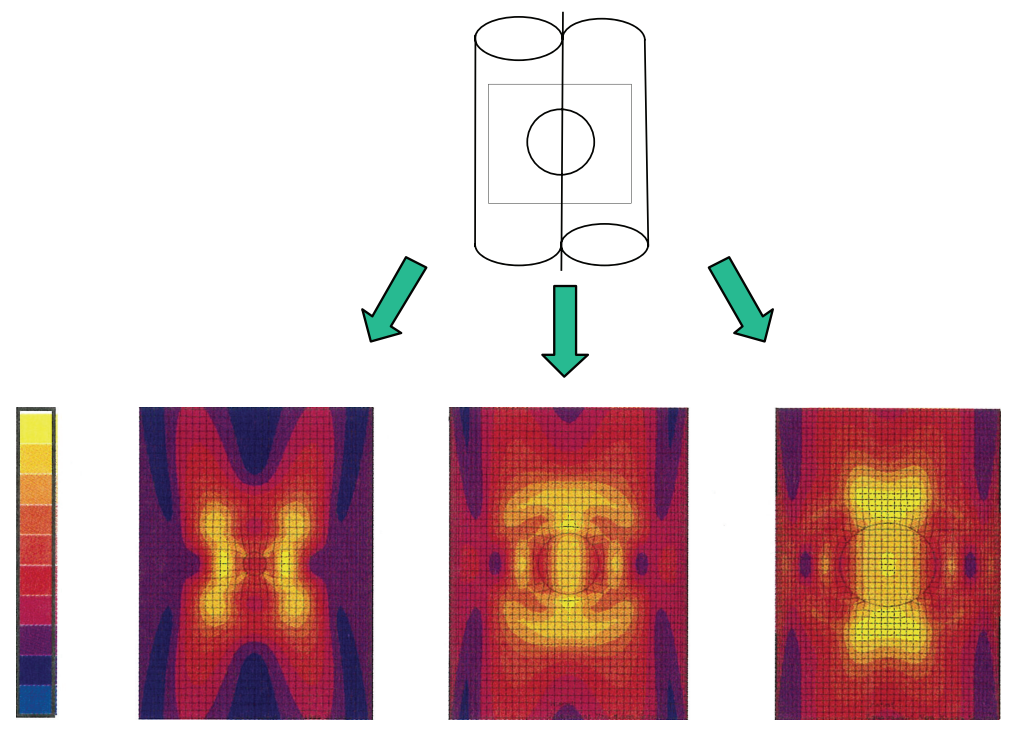

Figure 3: Von Mises stress distribution in the main tube near the branch joint at peak load.

Various empirical formulas have been proposed to date for the yield strength of a $T$-joint [1-3].

In [3]

$$
\frac{P_{\mathrm{cr}}}{\sigma_{Y} T^{2}}=6.43\left[1+4.60\left(\frac{d}{D}\right)^{2}\right] \text {, }
$$

In [2]

$$
\frac{P_{\mathrm{cr}}}{\sigma_{Y} T^{2}}=\frac{7.3}{1-0.833(d / D)},
$$

In [1]

$$
\frac{P_{\mathrm{cr}}}{\sigma_{Y} T^{2}}=2.14 \times f_{\beta} \cdot f_{\gamma} \cdot f_{n}
$$

In (3),

$$
f_{\beta}=0.96+4.7\left(\frac{d}{D}\right)^{2}, \quad f_{\gamma}=\left[\frac{d}{(2 T)}\right]^{0.2} .
$$

Furthermore, $f_{n}$ is a coefficient expressing the effect of the axial force on the main tube, $N$, where the following equation holds:

$$
f_{n}=1+0.3 n-0.3 n^{2}, \quad n=\frac{N}{\left(A \sigma_{Y}\right)},
$$

where A is the cross-sectional area of main tube. In Figure 4, $f_{n}=1$ because the axial force is $N=0$.

Figure 4 also shows the values predicted by the experimental formulas. In many cases, good agreement is found between the experimental formulas and the result of FEM analysis, but there are cases of large error, depending on the thickness $T$.

In (1) and (2), $P_{\mathrm{cr}} \propto T^{2}$ is used for yield strength $P_{\mathrm{cr}}$, while (3) uses $P_{\mathrm{cr}} \propto T^{1.8}$. To probe the relation between yield

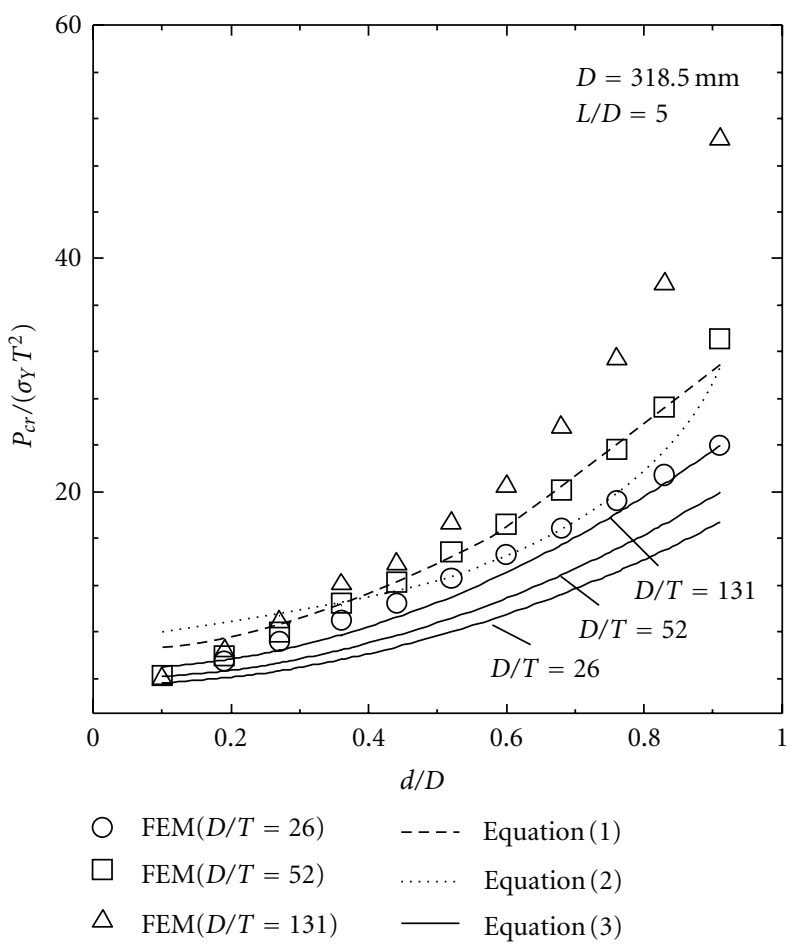

FIgURE 4: Relation between strength $P_{\text {cr }}$ and $d / D$ for $T$-joints.

strength $P_{c r}$ and main tube thickness $T$, the log-log graph of Figure 5 plots yield strength $P_{\text {cr }}$ against main tube thickness $T$ for $T$-joints with $d / D=0.1,0.27,0.44,0.60,0.76$, and 0.91 . As the figure shows, for any constant value of $d / D$, the yield strength $P_{\mathrm{cr}}$ is expressed by the exponent function in the form

$$
P_{\mathrm{cr}} \propto T^{\lambda}
$$




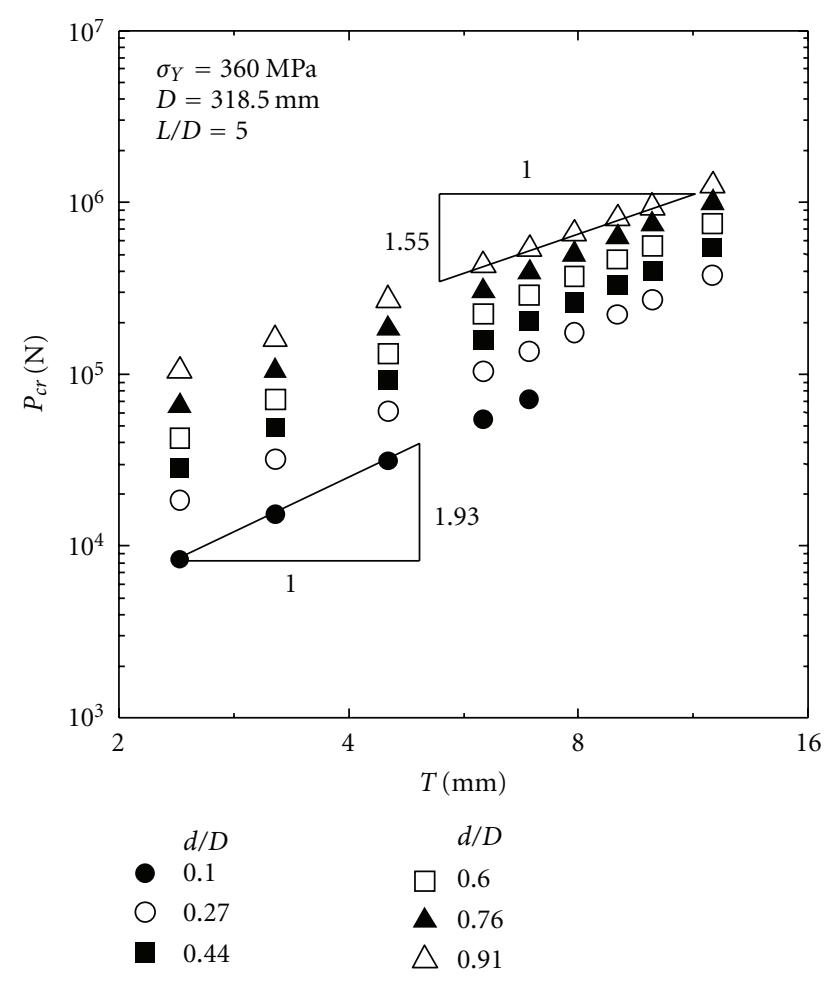

Figure 5: Relation between strength $P_{\text {cr }}$ and thickness $T$ of main tube plotted on a logarithmic scale.

where the exponent $\lambda$ depends on $d / D$, decreasing against $d / D$. In Figure $5, \lambda \cong 1.93$ holds for $d / D=0.1$ and $\lambda \cong 1.55$ for $d / D=0.91$.

In this study, common thickness of branch and main tubes $t=T=4.5 \mathrm{~mm}$ was used. Then, axial compressive buckling for branch tube did not occur. In this case, the yield strength $P_{\text {cr }}$ does not appreciably depend on the branch tube thickness $t$.

3.2. Crushing Load Behavior of Y-Joints. The angle between the branch and main tubes is $90^{\circ}$ in a $T$-joint, while it is expressed by $\theta$ in a $Y$-joint as shown in Figure 1(b).

As shown in Figure 6, the yield strength $\left.P_{c r}\right|_{Y}$ of a $Y$ joint is greater than that of a $T$-joint assuming the same values of $d / D$ and $T$. To estimate the yield strength of a $Y$ joint from that of a $T$-joint, we normally use the following approximation equation:

$$
\frac{\left.P_{\mathrm{cr}}\right|_{Y}}{P_{\mathrm{cr}}}=\frac{1}{\sin \theta}
$$

Figure 7 shows a comparison of the yield strength for a $Y$-joint predicted by the approximation equation (7) and the values obtained by FEM analysis. The figure shows that the prediction by (7) always lies in the safe side and agrees well with the data obtained by FEM analysis. In particular, in the case of a $Y$-joint with $\theta=45^{\circ}$, the values estimated by (7) agree well with the values from FEM analysis. However, in a $Y$-joint with $\theta<45^{\circ}$, the results of FEM analysis vary greatly with $d / D$, and the error of using (7) is larger.

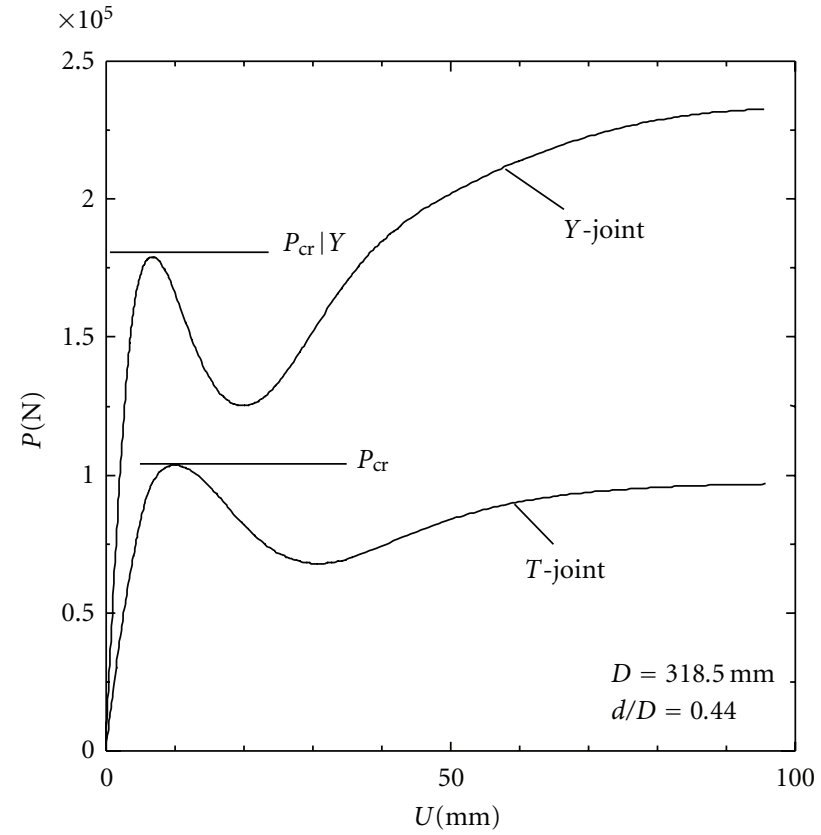

FIgURE 6: Load-displacement relationship in $T$ - and $Y$-joints.

One reason for this result is thought to be the existence of a force component $\left.P_{\mathrm{cr}}\right|_{Y} \cos \theta$ in the axial direction of the main tube due to the load $\left.P_{\mathrm{cr}}\right|_{Y}$. As discussed later in Section 3.3, the yield strength of a joint depends also on the axial force of the main tube, decreasing if the axial force is compressive and increasing if it is tensile. However, as has been pointed out by other researchers [4], in a $Y$-joint, the force component $\left.P_{\mathrm{cr}}\right|_{Y} \cos \theta$ in the axial direction of a main tube seems to act differently from the axial force acting on the main tube. Figure 8 shows the von Mises stress distribution at peak load for a $Y$-joint of $d / D=0.44$. (The schematic diagram in the left side of Figure 8 shows the positional relation between the contour diagram and the joint.) Because of the existence of a force component $\left.P_{\mathrm{cr}}\right|_{Y} \cos \theta$ in the axial direction, we have different stress values at the two top positions, with stress increasing in one and decreasing in the other. Furthermore, the joint geometry of branch and main tubes, which depends on the angle $\theta$ of the $Y$-joint, also affects the denting deformation, that is, the yield strength of the joint. Therefore, it is difficult to predict accurately the yield strength of a $Y$-joint from the yield strength of a $T$ joint. Note, however, that if $d / D$ is small, the effect of a force component $\left.P_{\mathrm{cr}}\right|_{Y} \cos \theta$ of an inclined load $\left.P_{\mathrm{cr}}\right|_{Y}$ in the axial direction of the main tube is considered small because the denting part of the main tube is mainly subject to the stress in the side part of the main tube as shown in Figure 3.

3.3. Effect of Axial Force along Main Tube. There is an axial force along the main tube in the actual structural object. Although the effect of the axial force along the main tube on the yield strength of a joint has been studied and design formulas have been proposed [1], the number of reports is few because the condition setting by experiment is difficult. 


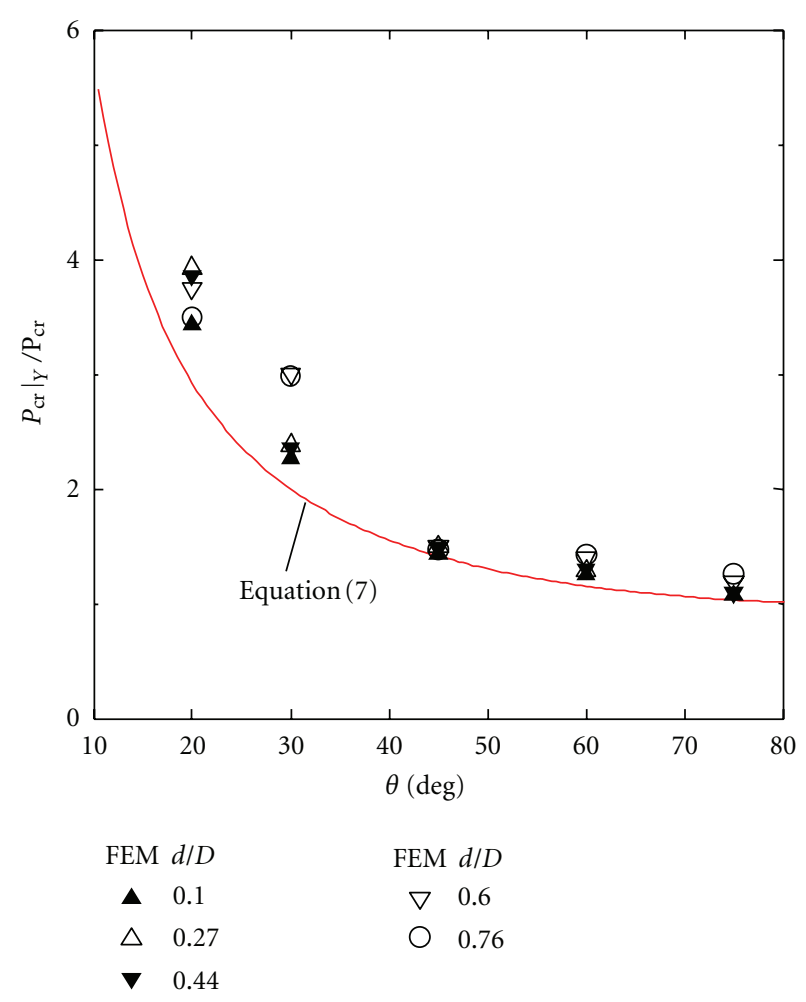

Figure 7: Strength $\left.P_{\mathrm{cr}}\right|_{Y}$ of $Y$-joints.

Therefore, we examined a wide range of conditions through numerical analyses.

Figures 9 and 10 show the ratio of yield strength $P_{\mathrm{cr}} /\left.P_{\mathrm{cr}}\right|_{N=0}$ for a $T$-joint and the ratio $\left.P_{\mathrm{cr}}\right|_{Y} /\left.P_{\mathrm{cr}}\right|_{Y, N=0}$ for a $Y$-joint with and without axial force along the main tube. As the figures show, the yield strength decreases if the axial force is compressive and increases if the axial force is tensile. The decrease and increase rates relate to the diameter ratio $d / D$ between branch and main tubes of the joint, increasing with $d / D$. Furthermore, from Figures 9 and 10, we can assume that the change rate of yield strength $\left.P_{\mathrm{cr}}\right|_{Y} /\left.P_{\mathrm{cr}}\right|_{Y, N=0}$ at a $Y$ joint and the change rate of yield strength $P_{\mathrm{cr}} /\left.P_{\mathrm{cr}}\right|_{N=0}$ at a $T$-joint are approximately the same for the same axial force and the same $d / D$, and the effect of the axial force along the main tube on yield strength $P_{\mathrm{cr}}$ is of similar magnitude in $T$ and $Y$-joints.

Figure 11 shows the relation between compressive load $P$ and branch tube displacement $U$ obtained from the numerical FEM analysis for a $T$-joint with and without axial force along the main tube; it is found that in comparison with the case without axial force along the main tube, the peak of compressive load $P$ in the branch tube appears later if the axial force along the main tube is tensile. Figure 12 shows the variation of the stress $\sigma_{x}$ in the axial direction at the top part of the main tube, owing to the indentation by the branch tube, as evaluated by the numerical FEM analysis. We focus on the top part of the main tube because a large stress appears in this part, as shown earlier in Figure 3, and this stress governs the yield strength $P_{\mathrm{cr}}$ of the joint. As Figure 12 shows, if the axial force is zero, the stress $\sigma_{x}$ in the direction of axial force, appearing at the top part due to the load $P$, is compressive, of which the absolute value increases with deformation and reaches yield stress as the compressive load reaches a peak (mark $\mathrm{A}$ in the figure). Furthermore, if the axial force is compressive, the compressive stress due to the axial force in the main tube is added to the compressive stress in the top part; the yield strength becomes small because the stress is high from the very early stage of deformation. On the other hand, if the axial force is tensile, the whole body of main tube is initially in the tensile state; if load is applied to the branch tube, compressive stress appears in the top part but is canceled by the initial tensile stress; a larger amount of load is needed for the deformation to reach the peak corresponding to yield strength $P_{\mathrm{cr}}$, and it would be natural to assume that the yield strength $P_{\mathrm{cr}}$ increases.

Equation (5) is proposed in [1] to predict the effect of axial force, and Figures 9 and 10 also show the values obtained from (5). It is found that the result of numerical analysis, which shows the same trend as (5), is always on the danger side and the difference is great especially if $d / D$ is large. For example, in a $T$-joint of $d / D=0.76$, the ratio between the yield strength obtained by FEM analysis and the yield strength with zero axial force $P_{\mathrm{cr}} /\left.P_{\mathrm{cr}}\right|_{N=0}$ is 0.600 if the axial force is $N /\left(A \sigma_{Y}\right)=-0.6$ while (5) gives 0.712 with the error $19 \%$ on the danger side. Therefore, Figures 9 and 10 also show the calculation using a modified formula:

$$
\begin{gathered}
f_{n}=1+0.35 n-0.35 n^{2} \\
n=\frac{N}{\left(A \sigma_{Y}\right)} .
\end{gathered}
$$

As the figure shows, the value calculated using (8) is nearly equal to the change rate of yield strength $P_{\mathrm{cr}} /\left.P_{\mathrm{cr}}\right|_{N=0}$ due to the compressive axial force for a joint of $d / D=0.44$.

3.4. Effect of Span Length of Main Tube. Figure 13 shows the variation of yield strength $P_{\mathrm{cr}}$ against the variation of span length $L$, which is obtained for the $T$-joints with various values of $d / D$ taking $L_{0}=1592.5 \mathrm{~mm}$ as a standard span length. Most approximation equations used to date do not specifically take into account the effect of span length $L$. However, the yield strength of a joint decreases with increasing span length, as shown in Figure 13. Although the effect is small if $d / D$ is small, the effect of span length $L$ on the yield strength of a joint is measurably great if $d / D$ is large. For example, against the joint of $d / D=0.91$, the yield strength decreases to about $73 \%$ for the joint of $L / L_{0}=2$.

The effect of span length on the yield strength of a joint can be discussed considering the bending stress in the main tube due to the load $P$ of the branch tube. First, it would be qualitatively understood that the effect of span is small when $d / D$ is small if one considers that the dent of the main tube is spatially limited to the side or the top area, respectively, for small or large $d / D$ and that the bending stress of the main tube is the greatest at the top area. Then, in the following, the effect of span length is quantitatively examined from the viewpoint of bending stress in the main tube due to the load $P$. 


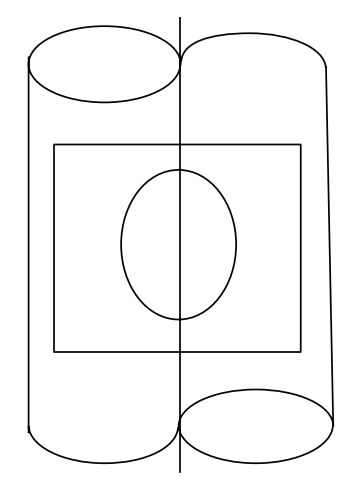

(a)

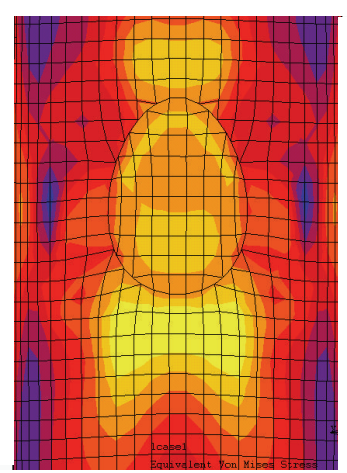

(b)

Figure 8: Von Mises stress distribution in the main tube surface at peak load for a $Y$-joint with $d / D=0.44$.

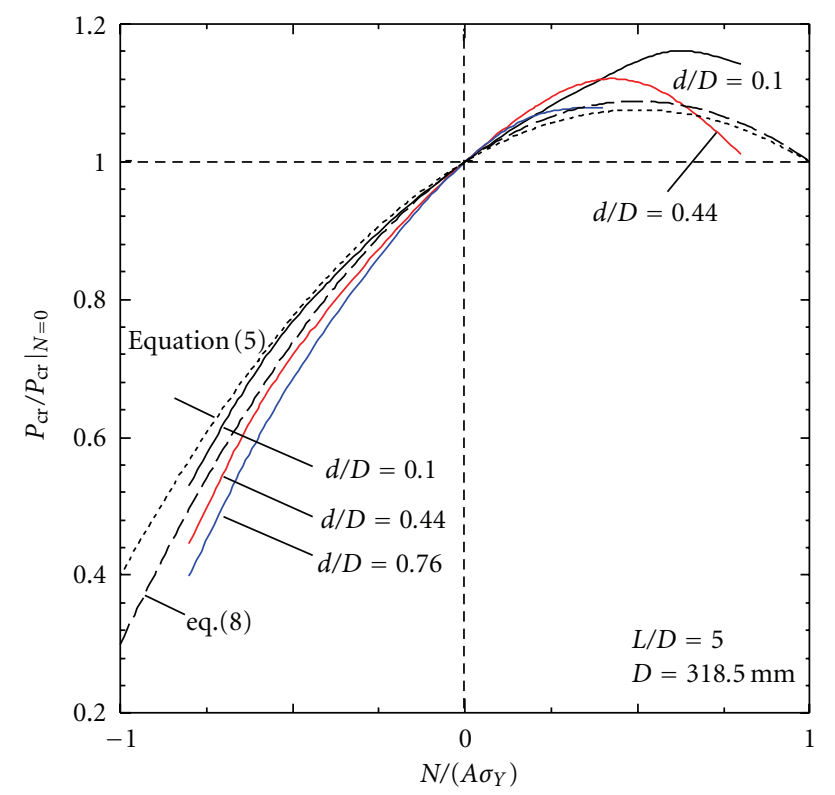

FIgURE 9: Strength $P_{\text {cr }}$ of $T$-joint with an axial force along the main tube.

Here, it is assumed that yield strength is reached in the joint when the stress $\sigma_{x}$ in the axial direction at the top area of the main tube reaches a certain critical value $\sigma_{\mathrm{cr}}$ of stress. Furthermore, it is assumed that the stress in the axial direction $\sigma_{x}$ is the sum of local stress $\sigma_{\text {loc }}$ due to the indentation caused by the branch tube and the bending stress $\sigma_{\text {glo }}$ at the top part of the main tube due to the load $P$ :

$$
\sigma_{\mathrm{cr}}=\sigma_{\mathrm{loc}}+\sigma_{\mathrm{glo}}
$$

Since the stress $\sigma_{\text {loc }}$ is not related to span length and is proportional to the yield strength $P_{\mathrm{cr}}$, it can be evaluated by using

$$
\sigma_{\mathrm{loc}}=\frac{k_{1}}{D T} P_{\mathrm{cr}}
$$

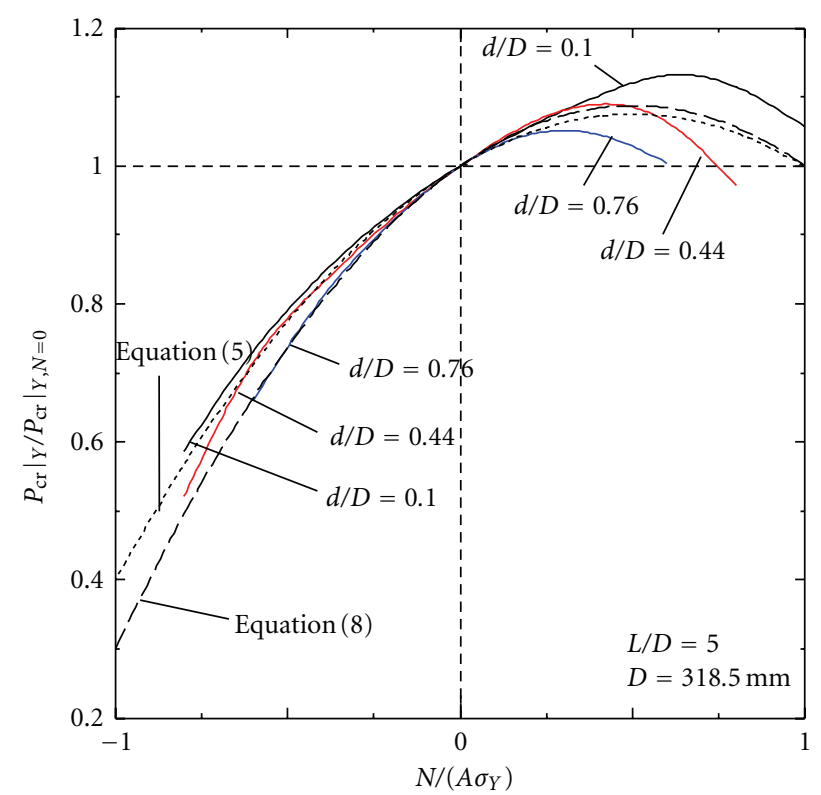

Figure 10: Strength $\left.P_{\text {cr }}\right|_{Y}$ of $Y$-joint with an axial force along the main tube.

Meanwhile, since the stress $\sigma_{\text {glo }}$ is bending stress due to the load $P$, it is proportional to span length $L$ and can be evaluated by using

$$
\sigma_{\mathrm{glo}}=\frac{k_{2}}{D^{2} T} P_{\mathrm{cr}} L
$$

In (10) and (11), $k_{1}$ and $k_{2}$ are unknown coefficients.

Using (9)-(11), we can estimate the yield strength for any span from the yield strength for the two different spans. For example, assuming the yield strength $\left.P_{\mathrm{cr}}\right|_{a}$ and $\left.P_{\mathrm{cr}}\right|_{b}$ for the joints with span lengths $L_{a}$ and $L_{b}$, the yield strength $P_{\mathrm{cr}}$ for a joint with an arbitrary span length $L$ is estimated as follows. 


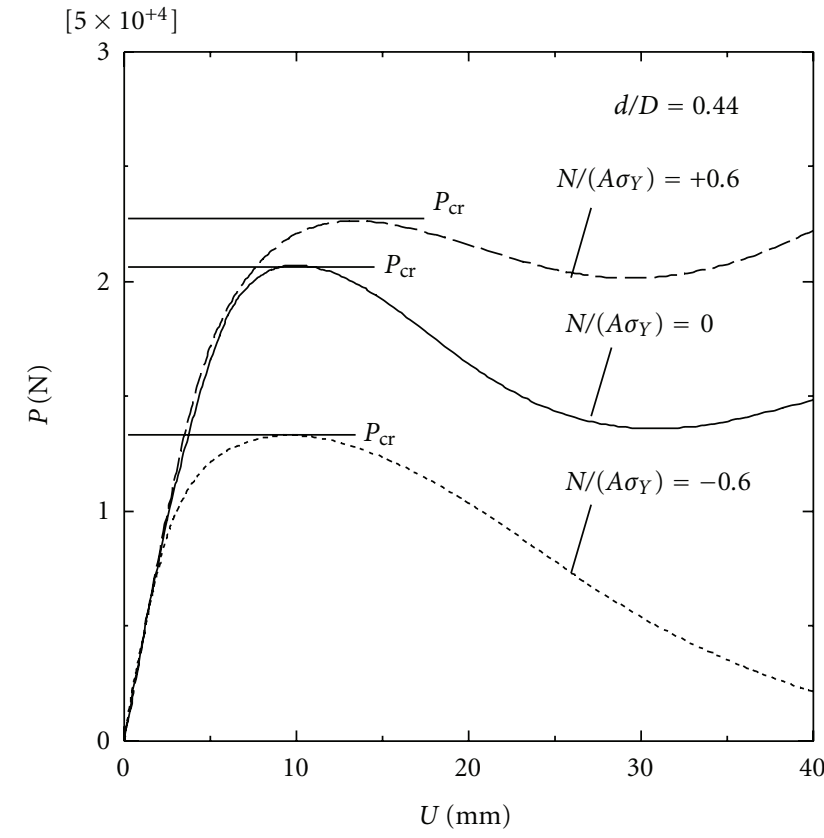

FIgURe 11: $P$ - $U$ curve for a joint without axial force along the main tube.

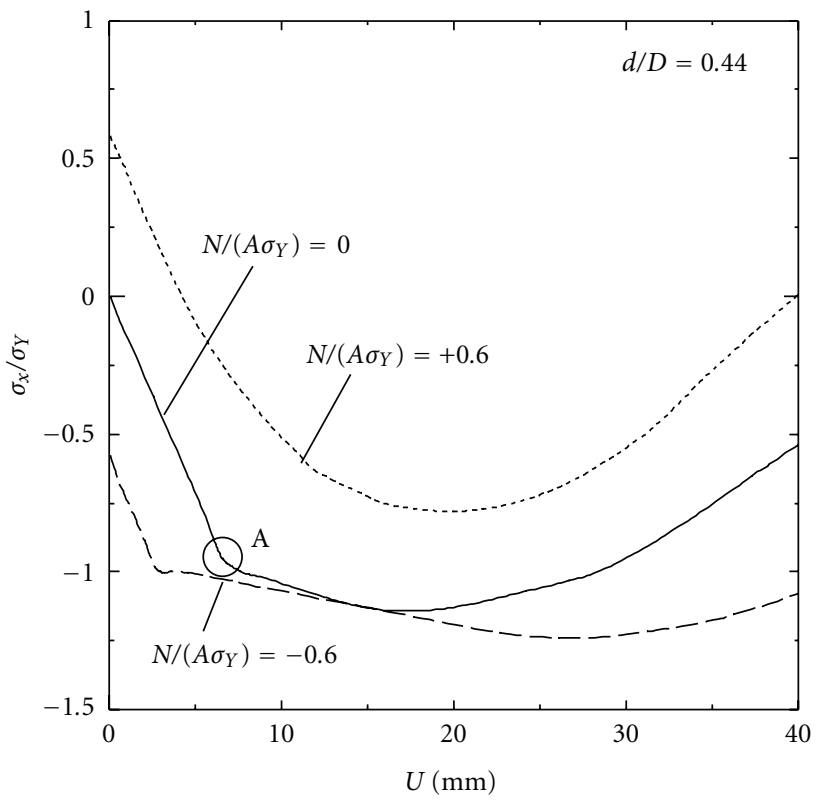

FIGURE 12: Change of stress $\sigma_{x}$ at the top part of the main tube.

From (9) to (11), the following equations hold for the joints with the span lengths $L_{a}, L_{b}$, and $L$ :

$$
\begin{gathered}
\sigma_{\mathrm{cr}}=\left.\frac{k_{1}}{D T} P_{\mathrm{cr}}\right|_{a}+\left.\frac{k_{2}}{D^{2} T} P_{\mathrm{cr}}\right|_{a} L_{a} \\
\sigma_{\mathrm{cr}}=\left.\frac{k_{1}}{D T} P_{\mathrm{cr}}\right|_{b}+\left.\frac{k_{2}}{D^{2} T} P_{\mathrm{cr}}\right|_{b L_{b}} \\
\sigma_{\mathrm{cr}}=\frac{k_{1}}{D T} P_{\mathrm{cr}}+\frac{k_{2}}{D^{2} T} P_{\mathrm{cr}} L .
\end{gathered}
$$

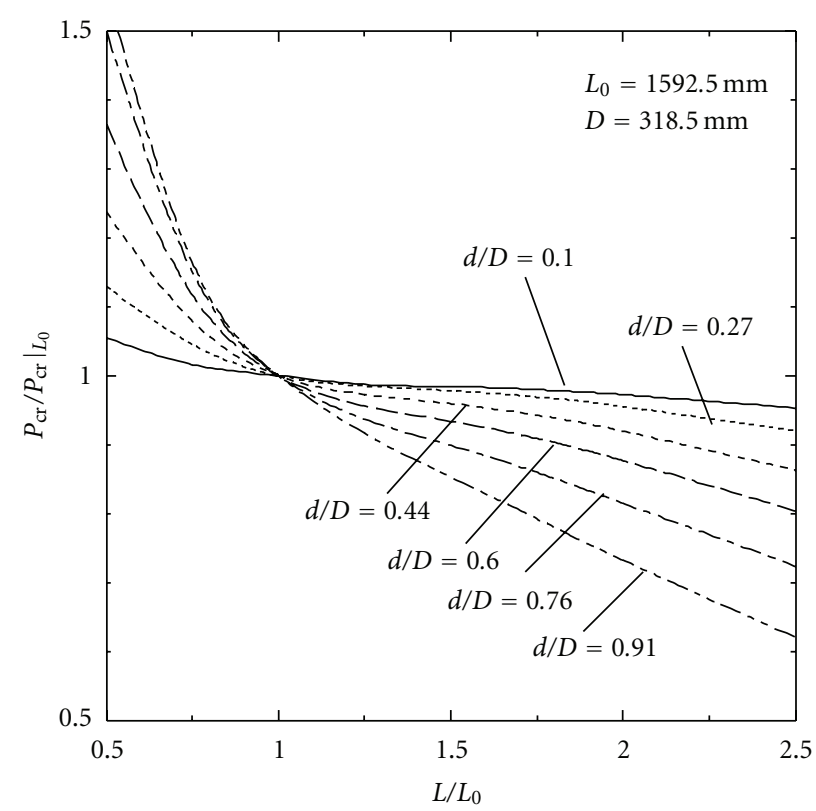

FIGURE 13: Effect of span length of main tube on strength of $T$-joint.

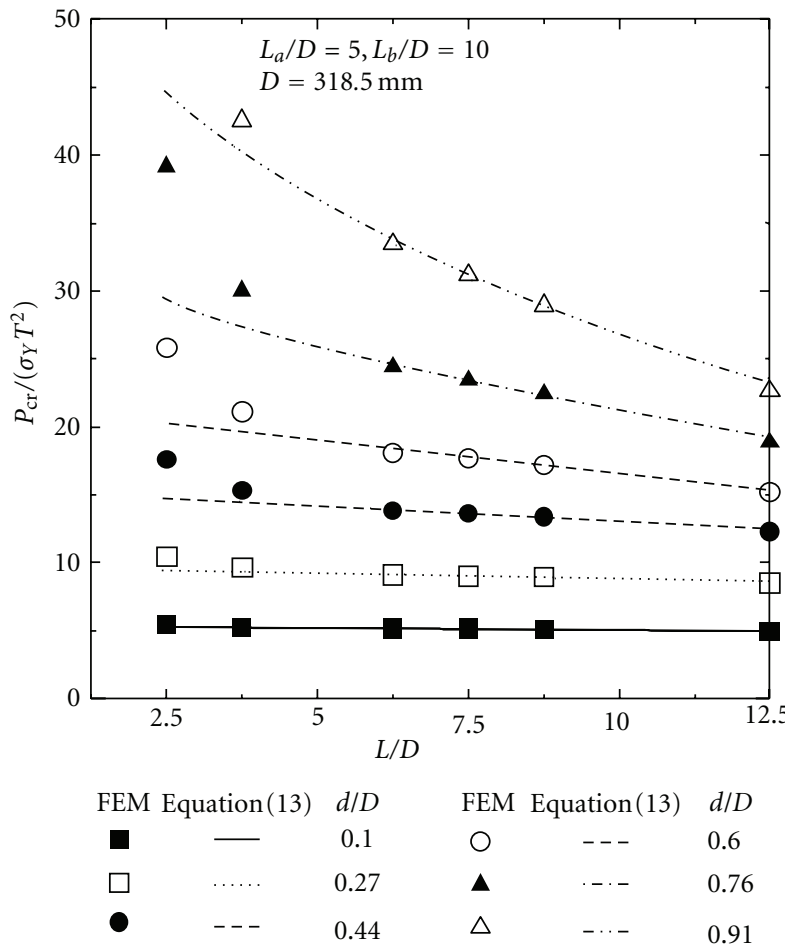

Figure 14: Prediction of (13).

From (12), from $\left.P_{\mathrm{cr}}\right|_{a}$ and $\left.P_{\mathrm{cr}}\right|_{b}$, we have an equation to estimate the yield strength $P_{\text {cr }}$ of a joint of length $L$ as follows:

$$
P_{\mathrm{cr}}=\frac{\left.\left.P_{\mathrm{cr}}\right|_{a} P_{\mathrm{cr}}\right|_{b}\left(L_{b}-L_{a}\right)}{\left.P_{\mathrm{cr}}\right|_{a} P_{\mathrm{cr}}\left(L-L_{a}\right)+\left.P_{\mathrm{cr}}\right|_{b}\left(L_{b}-L\right)} .
$$

Assuming $L_{a}=5 \mathrm{D}$ and $L_{b}=10 \mathrm{D}$, Figure 14 shows a comparison of the approximation by (13) and the numerical 
FEM analysis for the yield strength $P_{\text {cr }}$ of joints of length $L=$ $2.5 \mathrm{D}$ to $12.5 \mathrm{D}$. The figure shows that the two mostly agree with each other for a long span that satisfies, for example, $L / D>4$.

We can further examine the effect of span on yield strength through the effect of axial force. That is, we assume that the effect of the yield strength due to the bending stress $\sigma_{\text {glo }}$ in (11) is analogous to the effect of the axial force of the main tube; the ratio of axial force $n$ as used in (8) can be evaluated by using

$$
n=-\frac{k_{0}\left(\sigma_{\text {glo }}-\left.\sigma_{\text {glo }}\right|_{0}\right)}{\sigma_{Y}} .
$$

Here, $\left.\sigma_{\text {glo }}\right|_{0}$ is the bending stress at the standard span length $L_{0}$. By substituting (11) into (14), we obtain

$$
n=-\frac{k_{0} k_{2}}{D^{2} T} \frac{\left(P_{\mathrm{cr}} L-\left.P_{\mathrm{cr}}\right|_{0} L_{0}\right)}{\sigma_{Y}} .
$$

Here, $\left.P_{\mathrm{cr}}\right|_{0}$ is the yield strength of joint corresponding to the standard span length $L_{0}$. Furthermore, the coefficient $k_{0} k_{2}$ is assumed to be approximately $k_{0} k_{2} \cong 1 / 1.5$ based on investigating the application of the equation to various span lengths.

Therefore, using (8), we have an equation for $P_{\mathrm{cr}}$ as follows:

$$
\begin{aligned}
\frac{P_{\mathrm{cr}}}{\left.P_{\mathrm{cr}}\right|_{0}}= & 1-0.35\left(\frac{\left(P_{\mathrm{cr}} L-\left.P_{\mathrm{cr}}\right|_{0} L_{0}\right)}{1.5 D^{2} T \sigma_{Y}}\right) \\
& -0.35\left(\frac{\left(P_{\mathrm{cr}} L-\left.P_{\mathrm{cr}}\right|_{0} L_{0}\right)}{1.5 D^{2} T \sigma_{Y}}\right)^{2} .
\end{aligned}
$$

By solving this quadratic equation, we can estimate the yield strength $P_{\text {cr }}$ of a joint with a span length $L$ from the yield strength $\left.P_{\mathrm{cr}}\right|_{0}$ for the standard length $L_{0}$.

Figure 15 shows a comparison of the approximation by (16) and the numerical FEM analysis for the yield strength of a joint with length $L=2.5 \mathrm{D}$ to $12.5 \mathrm{D}$ assuming $L_{0}=5 \mathrm{D}$. It is shown that the two agree roughly with each other for any long span satisfying, for example, $L / D>4$.

Meanwhile, although the span length clearly influences yield strength, the issue of how to define the standard span length for design of an actual structure and apply it in the design equations remains. These issues are left for future study.

\section{Conclusions}

Although the yield strength of steel joints depends not only on buckling and plastic yield but also on the brittle failure of welded parts and punching shear, we focused on only the yield strength originating from buckling and plastic yield and performed analysis with a general-purpose analysis software using the finite element method on the yield strength of $T$ and $Y$-joints of steel pipes in the range of diameter ratio $d / D=0.1$ to 0.91 and span length $L / D=2.5$ to 12.5 . As a result, the following conclusions were obtained.

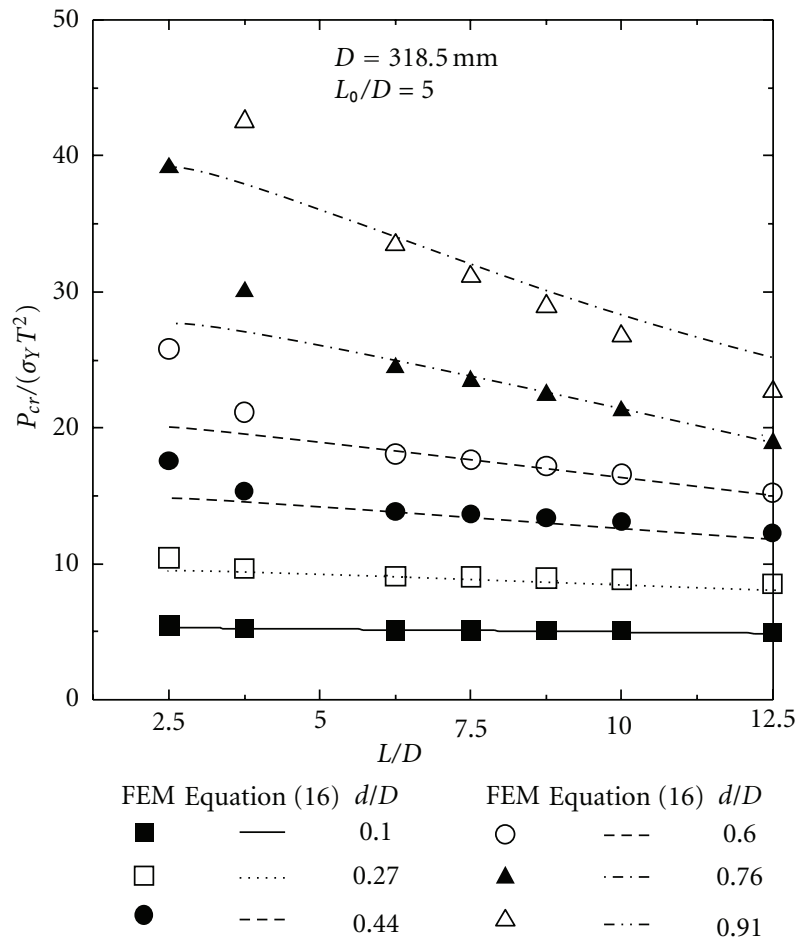

Figure 15: Prediction from (16).

(1) When the diameter ratio $d / D$ is kept constant between branch and main tubes, the yield strength $P_{\text {cr }}$ depends on the power function of the thickness $T$ of the main tube, $P_{\text {cr }} \propto T^{\lambda}$, where the power $\lambda$ depends on $d / D$, decreasing against $d / D . \lambda \cong 1.93 \sim$ 1.55 holds in the range $d / D=0.1 \sim 0.91$.

(2) The yield strength of a joint decreases as span length increases. Furthermore, although the effect is small if $d / D$ is small, the effect of span length $L$ on the yield strength of a joint is large if $d / D$ is large. We can examine the effect of span length on the yield strength of a joint by considering the bending stress of the main tube due to the load $P$.

(3) The yield strength of a joint decreases due to the axial compressive force of the main tube, and the magnitudes of the effect are similar in $T$ - and $Y$ joints. The effect of axial force can be understood considering that the dent of the main tube is governed mainly by the axial stress $\sigma_{x}$ due to the load $P$ and the axial stress $\sigma_{x}$ is compressive.

(4) Although the relation $\left.P_{\mathrm{cr}}\right|_{Y}<P_{\mathrm{cr}} / \sin \theta$ always holds between the yield strength $\left.P_{\mathrm{cr}}\right|_{Y}$ of a $Y$-joint and that of a $T$-joint for the same value of $d / D$, the equation $\left.P_{\mathrm{cr}}\right|_{Y} \cong P_{\mathrm{cr}} / \sin \theta$ approximately holds. In particular, the error is small at $\theta=45^{\circ}$ or when $d / D$ is small. 


\section{References}

[1] Architectural Institute of Japan, Recommendations for the Design and Fabrication of Tubular Truss Structures in Steel, chapter 4, 2002.

[2] N. Akiyama, M. Yajima, H. Akiyama, and F. Ohtake, "Experimental study on strength of joints in steel tubular structures," Journal of Society of Steel Construction, vol. 10, no. 102, pp. 3768, 1974 (Japanese).

[3] Y. Makino, Y. Kurobane, and Y. Mitsui, "Ultimate strength of tubular joints for local failures due to compression braces," Transactions of Architectural Institute of Japan, vol. 251, pp. 45$55,1977$.

[4] K. Washio, T. Togo, and Y. Mitsui, "Experimental research on local failure of chord in tubular truss joints," Transactions of Architectural Institute of Japan, vol. 140, pp. 55-62, 1967.

[5] H. Kanatani, "Experimental study of welded tubular connection (Part 1)," Transactions of Architectural Institute of Japan, vol. 108, pp. 31-39, 1965 (Japanese).

[6] E. M. Dexter and M. M. K. Lee, "Static strength of axially loaded tubular K-joints. I: behavior," Journal of Structural Engineering, vol. 125, no. 2, pp. 194-201, 1999. 

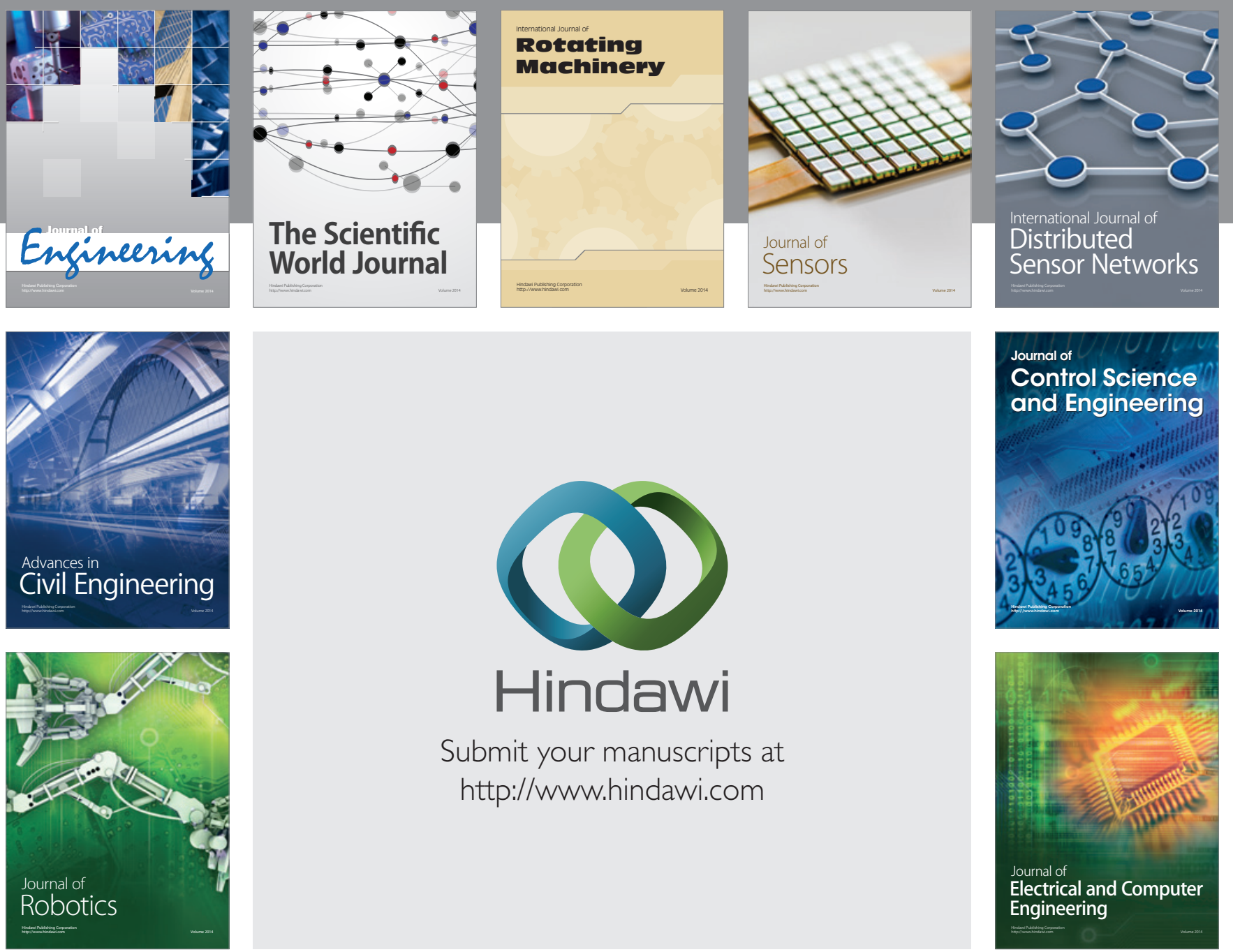

Submit your manuscripts at

http://www.hindawi.com
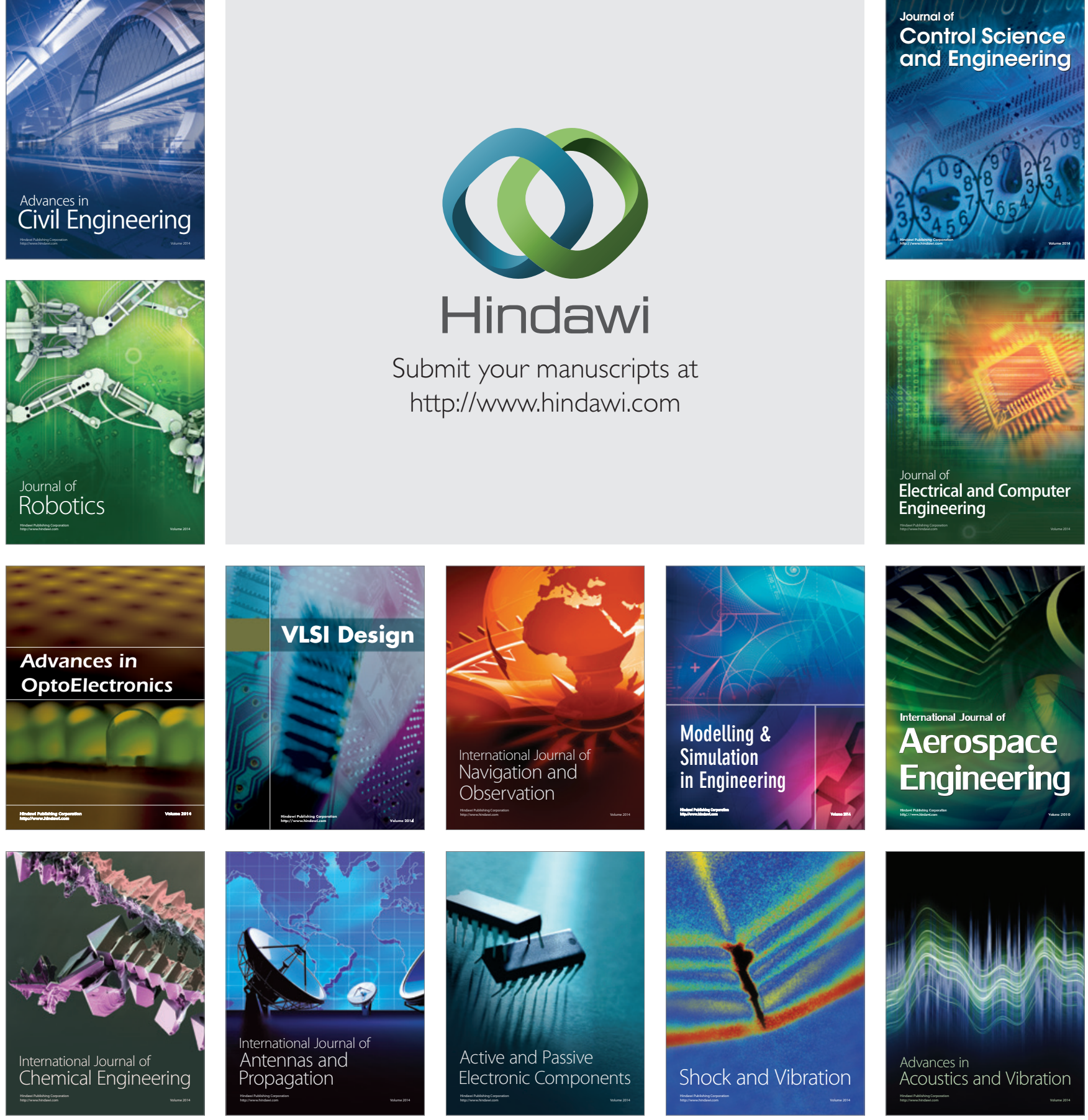